\title{
Surface photometry of radio loud elliptical galaxies from the B2 sample ${ }^{\star, \star \star}$
}

\author{
J.I. González-Serrano ${ }^{1}$ and R. Carballo ${ }^{1,2}$ \\ 1 Instituto de Física de Cantabria, CSIC-Universidad de Cantabria, Facultad de Ciencias, E-39005 Santander, Spain \\ 2 Departamento de Física Moderna, Universidad de Cantabria, Facultad de Ciencias, E-39005 Santander, Spain
}

Received July 8; accepted November 16, 1999

\begin{abstract}
V$-band CCD imaging is presented for 72 galaxies from the B2 radio sample (Colla et al. 1975; Fanti et al. 1978), with redshifts up to 0.2 and radio powers $P_{408}=$ $10^{23}-10^{26.5} \mathrm{~W} \mathrm{~Hz}^{-1}$. According to the morphology on the optical images 57 galaxies are classified as ellipticals, 6 as spirals and 7 as irregular. Surface photometry of the sample of ellipticals was obtained fitting ellipses to the light distribution. The light profile of these galaxies generally follows a de Vaucouleurs law, although in three cases the profiles show large excesses relative to the $r^{1 / 4}$ law at large radii. The fitted $\mu_{\mathrm{e}}$ and $r_{\mathrm{e}}$ parameters for the de Vaucouleurs galaxies are given in the paper. Three of the ellipticals show a bright nucleus. One of them is a known broad line radio galaxy (B2 $1833+32$ ) and the remaining two are Markarian galaxies, classified in the literature as BL Lac objects (B2 1101+38 and B2 1652+39). The radial profiles for ellipticity, position angle, and $B_{4}$ term of the Fourier analysis are presented in the paper, and the morphological peculiarities of the ellipticals are described, including the presence of shells, tails, nuclear dust, isophote twisting, off-centering, and boxiness or diskness of the isophotes. Only one of the galaxies in this work is included in the subsample of B2 radio galaxies with welldefined jets (Parma et al. 1987). In this sense the present sample complements the sample of 24 radio galaxies with well-defined radio jets in Parma et al. for which a similar study was presented in González-Serrano et al. (1993).
\end{abstract}

The irregular galaxy B2 0916+33 appears to be misclassified, and we suggest that the right identification of the radio source is a nearby point like object with $V=18.45 \mathrm{mag}$. The spiral galaxy associated with B2 $1441+26$ is also misclassified. A point-like optical object

Send offprint requests to: J.I. González-Serrano, e-mail: gserrano@ifca.unican.es

* Table 4 is only available in electronic form at the CDS via anonymous ftp to cdsarc.u-strasbg.fr (130.79.128.5) or via http://cdsweb.u-strasbg.fr/Abstract.html

** Complete Figure 1 and Figure 2 are only available at http://www.edpsciences.org with $V=18.88 \mathrm{mag}$, located at $\sim 36$ arcsec from the original identification and coincident with the radio core is the most probable counterpart.

Key words: galaxies: active — galaxies: fundamental parameters — galaxies: photometry — radio continuum: galaxies

\section{Introduction}

Most of the radio sources in the luminosity range $P_{408} \sim$ $10^{23}-10^{25} \mathrm{~W} \mathrm{~Hz}^{-1}\left(H_{0}=75 \mathrm{~km} \mathrm{~s}^{-1} \mathrm{Mpc}^{-1}\right.$ and $q_{0}=0$ is assumed throughout the paper) and at low redshift are found in elliptical galaxies. These galaxies form the population referred to as "Low Luminosity Radio Galaxies" (hereafter LLRGs), within the class of active galactic nuclei. LLRGs have lower radio luminosity than "Powerful Radio Galaxies" (hereafter PRGs) - whose radio power is similar to that of radio quasars-, but larger radio luminosity than starburst galaxies. Optical studies of the elliptical hosts of LLRGs show that their photometric parameters resemble the class of brightest cluster members (hereafter BCMs), exhibiting similar absolute magnitudes in average, shallow light profiles, and presenting extended envelopes (cD type) for a significant fraction of sources (Lilly \& Prestage 1987; Owen \& Laing 1989; Smith \& Heckman 1989a, 1989b; González-Serrano et al. 1993). Regarding the local environment, Heckman et al. (1985) and González-Serrano et al. (1993) found that LLRGs were located in higher density regions that radio quiet ellipticals, considering a radius of $133 \mathrm{kpc}$ around the radio galaxy. Heckman et al. (1985) concluded from this result that galaxy interactions could foster the nuclear activity. Alternatively, Ledlow \& Owen (1995) claimed from their study of LLRGs in Abell clusters that the radio detection 
rate was influenced by the optical properties, i.e. the optical luminosity, rather than the galaxy density around the radio galaxy (hereafter $R G$ ).

Quantitative analysis of the morphological structure of the galaxy isophotes, obtained from surface photometry, has revealed morphological peculiarities in LLRGs which are likely related to gravitational interaction and merging processes (Colina \& Pérez-Fournon 1990; González-Serrano et al. 1993). These characteristics include strong isophote twisting, off-centering, excesses from a de Vaucouleurs light profile at large radii and the presence of features like shells, tails and bridges. This type of peculiarities had been previously observed in PRGs $\left(P>10^{25}-10^{25.5} \mathrm{~W} \mathrm{~Hz}^{-1}\right.$, Heckman et al. 1986; Smith \& Heckman 1989b), where they are generally more significant. PRGs differ from LLRGs in other respects. The former typically have an FR II (Fanaroff \& Riley 1974) radio morphology (whereas the latter are generally FR I), are located in poorer local environments, their Hubble type and surface brightness profiles are more diverse, and they have stronger emission lines and a larger fraction of systems showing dust patches (Heckman et al. 1986). Heckman et al. (1986) argued that PRGs could originate from galaxy mergers involving at least a dynamically cold gas-rich system (a disk galaxy) whereas that LLRGs could be fueled by accretion of their own atmospheres or of an intracluster or intragroup gaseous medium. Smith \& Heckman (1989b) suggested that mergers could be common to both classes, but that mergers for LLRGs would occur between ellipticals, and the lack of a cool interstellar medium would prevent the formation of the strong tidal perturbations shown in PRGs.

González-Serrano et al. (1993) reported an exhaustive photometric study of a sample of 24 LLRGs from the B2 sample (Colla et al. 1975; Fanti et al. 1978), with the common characteristic of presenting a well-defined radio jet in Parma et al. (1987). Various general properties were established for this sample, like the shape of the light profiles, the absolute magnitudes, local environment, incidence of isophote peculiarities and fraction of systems undergoing gravitational interaction/merging. It was concluded that, at least among galaxies with radio jets, the local galaxy density and the interactions likely play an important role in the production of this type of radio sources. One question arising from this study was whether LLRGs with and without a well-defined jet have similar environments and optical properties. The answer will help to understand the origin of the different kinds of radio sources and the relation of radio morphology with the environment.

The present paper reports surface photometry for 72 B2 galaxies (including some spirals and irregulars), based on $V$-band CCD images, and complements our previous optical studies of B2 galaxies with well-defined radio jets. The following results are presented: (i) $V$-band contour plots of the galaxies and qualitative morphological clas- sification, (ii) surface brightness profiles of the elliptical galaxies and de Vaucouleurs fits, obtaining the parameters $\mu_{\mathrm{e}}$ and $r_{\mathrm{e}}$, (iii) profiles of the isophote parameters ellipticity, position angle and $B_{4}$, and (iv) description of the isophote peculiarities of the galaxies, relative to an elliptical model, as well as other morphological features such as tails, shells and/or nuclear dust. The relation of the optical properties with radio morphology for the complete B2 sample will be presented in a forthcoming paper.

\section{The sample}

The B2 sample of LLRGs comprises the samples by Colla et al. (1975, hereafter C75) and Fanti et al. (1978, herafter F78). Both samples are optically complete down to the limiting magnitudes of $m_{\mathrm{pg}}=15.7$ for C75, known as the "bright" sample, and $m_{V}=16.5$ for F78, known as the "faint" sample. A few galaxies on the "faint" sample have $V$ magnitudes in the range $16.5-17$ mag. On the radio side the two samples are complete down to $0.25 \mathrm{Jy}$ at $408 \mathrm{MHz}$, although fainter radio galaxies are listed down to $0.2 \mathrm{Jy}$. The average redshift for the "bright" sample is 0.03 and the average radio power $P_{408} \sim 10^{24.2} \mathrm{~W} \mathrm{~Hz}^{-1}$. The same parameters for the "faint" sample are 0.09 and $10^{25.1} \mathrm{~W} \mathrm{~Hz}^{-1}$. Whereas that the "bright" sample includes galaxies of all morphological types, only those classified as ellipticals were included in the F78 "faint" sample.

High quality radio maps for most of the galaxies were obtained in a systematic way by the Bologna group using VLA configurations A,B and/or C, or their combination (Parma et al. 1986; de Ruiter et al. 1986; Fanti et al. 1986, 1987; Morganti et al. 1987). The sample was also subject to systematic studies of their radio cores (Feretti et al. 1984; Giovannini et al. 1988) and their IR properties (Impey \& Gregorini 1993). After removing eight misclassified galaxies the combined B2 sample comprised 115 galaxies (Fanti et al. 1987, hereafter F87). Parma et al. (1987) analysed from the available radio maps from the Bologna group and the literature the presence of radio jets, obtaining a subsample of $43 \mathrm{~B} 2$ radio galaxies with jets and a smaller subsample of 11 sources with ill-defined radio jets. The radio sources observed for this work include all but one (B2 1426+25) of the 72 galaxies in the B2 sample not classified by Parma et al. (1987) as having a well-defined jet. Surface photometry of a subset of $24 \mathrm{~B} 2$ radio galaxies with jets was previously reported by our group (González-Serrano \& Pérez-Fournon 1989, 1991, 1992; González-Serrano et al. 1993, hereafter Papers I-IV) and sources with jets in Parma et al. (1987) were deliberately excluded for the present work. An exception was the galaxy B2 $0326+39$ (not included in Papers I-IV), which was observed with the new sources due to scarcity of other targets at the right ascensions observable during the runs. Together with the data in Papers I-IV we have now obtained $V$-band optical CCD imaging for 83 per cent $(96 / 115)$ of the 
Table 1. Source list

\begin{tabular}{|c|c|c|c|c|c|c|c|}
\hline B2 name & Other names & R.A.(1950) & $\operatorname{Dec}(1950)$ & $\log P_{408}$ & $z$ & Radio $\operatorname{morph}^{a}$ & $\operatorname{Ref}^{b}$ \\
\hline $0116+31$ & $4 \mathrm{C} 31.04$ & 011647.1 & 315507 & 25.31 & 0.0592 & VLA calib & \\
\hline $0120+33$ & NGC 507 & 012050.7 & 325945 & 23.47 & 0.0164 & no jet & \\
\hline $0149+35$ & NGC 703 & 014950.0 & 355420 & 23.17 & 0.0160 & jet & \\
\hline $0207+38$ & NGC 828 & 020707.1 & 385722 & 23.26 & 0.0181 & no jet & \\
\hline $0209+37$ & & 020952.8 & 373454 & 23.36 & 0.0177 & - & \\
\hline $0222+36$ & & 022223.9 & 365657 & 23.77 & 0.0327 & no jet & \\
\hline $0258+35$ & 4C 34.09, NGC 1167 & 025835.6 & 350031 & 24.20 & 0.0160 & no jet & \\
\hline $0326+39$ & UGC 2755 & 032606.5 & 393712 & 24.26 & 0.0243 & jet & \\
\hline $0331+39$ & $4 \mathrm{C} 39.12$ & 033101.1 & 391125 & 24.05 & 0.0202 & no jet & \\
\hline $0648+27$ & & 064854.2 & 273117 & 23.87 & 0.0409 & no jet & \\
\hline $0708+32$ & & 070833.6 & 322337 & 24.34 & 0.0672 & no jet & \\
\hline $0722+30$ & & 072227.8 & 300320 & 23.35 & 0.0191 & jet & \\
\hline $0800+24$ & & $\begin{array}{lll}08 & 00 & 16.2\end{array}$ & 244906 & 23.99 & 0.0433 & no jet & \\
\hline $0828+32$ & $4 \mathrm{C} 32.25$ & 082820.6 & $32 \quad 2937$ & 25.27 & 0.0507 & jet & \\
\hline $0838+32$ & $4 \mathrm{C} 32.26$ & 083806.6 & 323543 & 25.17 & 0.0680 & no jet & \\
\hline $0843+31$ & & 084338.3 & 313709 & 24.43 & 0.0665 & no jet & \\
\hline $0916+33$ & & 091652.5 & 330843 & - & 0.0500 & misclass & \\
\hline $0922+36$ & $4 \mathrm{C} 36.14$ & 092234.3 & 364005 & 25.60 & 0.1125 & no jet & \\
\hline $0924+30$ & IC 2476 & 092455.1 & 301215 & 24.30 & 0.0266 & no jet & \\
\hline $0944+39$ & & 094446.3 & 394558 & 23.94 & 0.0410 & - & \\
\hline $1003+26$ & & 100349.4 & 260924 & 24.84 & 0.1165 & no jet & \\
\hline $1003+35$ & $3 \mathrm{C} 236$ & 100305.4 & 350847 & 26.01 & 0.0989 & no jet & \\
\hline $1037+30$ & $4 \mathrm{C} 30.19$ & 103742.7 & 301338 & 25.20 & 0.0908 & no jet & \\
\hline $1039+27$ & & 103959.0 & 270621 & 23.60 & 0.0203 & - & \\
\hline $1101+38$ & & 110140.6 & 382843 & 24.22 & 0.0300 & no jet & \\
\hline $1102+30$ & & 110239.7 & 302553 & 24.92 & 0.0720 & jet & \\
\hline $1106+26$ & NGC 3534 & 110614.1 & 265251 & 23.49 & 0.0219 & - & \\
\hline $1113+24$ & & 111324.0 & 245724 & 24.66 & 0.1021 & no jet & \\
\hline $1141+37$ & $4 \mathrm{C} 37.32$ & 114149.7 & $37 \quad 2511$ & 26.06 & 0.1140 & no jet & \\
\hline $1144+35$ & & 114445.5 & 351749 & 24.34 & 0.0630 & ji & \\
\hline $1204+24$ & & 120434.3 & 241106 & 24.43 & 0.0769 & jet & \\
\hline $1204+34$ & & 120500.4 & 340921 & 25.02 & 0.0788 & no jet & \\
\hline $1217+29$ & NGC 4278 & 121736.6 & 293329 & 21.61 & 0.0021 & no jet & \\
\hline $1251+27$ & $3 \mathrm{C} 277.3$ & 125146.2 & 275351 & 25.89 & 0.0857 & no jet & \\
\hline $1256+28$ & NGC 4869 & 125658.7 & 281051 & 24.07 & 0.0224 & no jet & \\
\hline $1257+28$ & NGC 4874 & 125711.3 & 281350 & 23.66 & 0.0239 & no jet & 10 \\
\hline $1300+32$ & & 130054.4 & 320610 & 25.59 & 0.1647 & no jet & \\
\hline $1303+31$ & & 130328.3 & 311020 & 25.28 & 0.1816 & no jet & 1 \\
\hline $1316+29$ & 4C 29.47 & 131643.0 & 295420 & 25.44 & 0.0728 & jet & \\
\hline $1317+33$ & NGC 5098 & 131755.8 & 332419 & 23.71 & 0.0363 & no jet & \\
\hline $1318+34$ & & 131817.0 & 342400 & 23.28 & 0.0232 & no jet & 2 \\
\hline $1339+26$ & 4C 26.41, UGC 8669 & 133930.7 & 263720 & 25.08 & 0.0753 & no jet & \\
\hline $1346+26$ & $4 \mathrm{C} 26.42$ & 134634.0 & 265028 & 25.32 & 0.0633 & no jet & 1,11 \\
\hline $1347+28$ & & 134756.3 & 283134 & 24.66 & 0.0724 & jet & 1 \\
\hline $1358+30$ & & 135833.4 & 303621 & 25.26 & 0.1104 & no jet & \\
\hline $1422+26$ & & 142226.5 & 265102 & 24.65 & 0.0370 & jet & 1 \\
\hline $1430+25$ & $4 \mathrm{C} 25.46$ & 143026.9 & 250828 & 25.19 & 0.0813 & no jet & 1 \\
\hline $1441+26$ & & 144153.9 & 261351 & - & 0.0621 & misclass & 1 \\
\hline $1447+27$ & & 144717.8 & 275913 & 23.71 & 0.0306 & no jet & \\
\hline $1455+28$ & $4 \mathrm{C} 28.38$ & 145545.5 & 284416 & 25.76 & 0.1411 & no jet & 1 \\
\hline $1457+29$ & & 145734.4 & 291523 & 25.42 & 0.1460 & no jet & 1 \\
\hline $1502+26$ & $3 \mathrm{C} 310, \mathrm{VV} 204 \mathrm{~b}$ & 150246.9 & 261234 & 26.04 & 0.0535 & no jet & 12 \\
\hline
\end{tabular}


Table 1. continued

\begin{tabular}{lccccccr} 
B2 name & Other names & R.A.(1950) & Dec(1950) & $\log P_{408}$ & $z$ & Radio morph $^{a}$ & Ref $^{b}$ \\
\hline $1511+26$ & 3C 315 & 151130.8 & 261839 & 26.31 & 0.1076 & no jet & 1 \\
$1512+30$ & & 151159.4 & 301954 & 24.60 & 0.0931 & no jet & $2 \mathrm{~F}$ \\
$1527+30$ & & 152743.3 & 305249 & 25.00 & 0.1143 & no jet & 1 \\
$1557+26$ & & 155745.9 & 260451 & 23.90 & 0.0442 & no jet & $2 \mathrm{~F}$ \\
$1609+31$ & & 160942.3 & 311041 & 24.52 & 0.0944 & no jet & 1 \\
$1610+29$ & NGC 6086 & 161035.4 & 293642 & 23.71 & 0.0313 & no jet & 1 \\
$1615+32$ & 3C 332 & 161546.8 & 322949 & 26.32 & 0.1520 & jet & 1 \\
$1615+35$ & 4C 30.40, NGC 6109 & 161549.0 & 350732 & 24.88 & 0.0296 & ji & 6 \\
$1621+38$ & NGC 6137 & 162116.9 & 380217 & 24.16 & 0.0310 & no jet & 1 \\
$1626+39$ & 3C 338, NGC 6166 & 162655.6 & 393936 & 25.43 & 0.0303 & jet & 1 \\
$1652+39$ & 4C 39.49 & 165211.8 & 395025 & 24.51 & 0.0337 & no jet & 2 \\
$1657+32$ & 4C 32.52 & 165708.6 & 323404 & 24.92 & 0.0631 & no jet & 1 \\
$1658+32$ & & 165818.7 & 323937 & 25.03 & 0.1020 & no jet & 1 \\
$1726+31$ & 3C 357 & 172627.4 & 314825 & 26.48 & 0.1670 & no jet & 1 \\
$1833+32$ & 3C 382 & 183312.0 & 323918 & 25.89 & 0.0586 & jet & 1 \\
$1855+37$ & & 185554.3 & 375627 & 24.60 & 0.0552 & no jet & 1 \\
$2240+29$ & & 224018.3 & 292749 & 23.38 & 0.0254 & - & - \\
$2257+25$ & & 225745.5 & 254941 & 23.45 & 0.0242 & - & \\
$2320+32$ & & 232043.4 & 321509 & 23.02 & 0.0177 & - & - \\
$2335+30$ & & 2354.6 & 302552 & 20.52 & 0.0008 & & \\
\hline
\end{tabular}

${ }^{a}$ ji=ill-defined radio jet (Parma et al. 1987).

${ }^{b}$ References for radio maps: (1). Series of VLA observations of B2 LLRGs by the Bologna group (Parma et al. 1986; de Ruiter et al. 1986; Fanti et al. 1986; Fanti et al. 1987); (2). Sources studied in those papers but without published radio maps, since the sources were only slightly resolved; $(2 \mathrm{~F})$. Sources in the previous category which are resolved on the FIRST images; (Faint Images of the Radio Sky at Twenty Centimeters, White et al. 1997); (3). Bridle et al. 1991; (4). Ekers et al. 1981; (5). Barthel et al. 1985; (6). Machalsky \& Condon 1985; (7). Schilizzi et al. 1983; (8). van Breugel et al. 1985; (9). O'Dea \& Owen 1985; (10). Feretti \& Giovannini 1985; (11). van Breugel et al. 1984; (12). van Breugel \& Jägers 1982.

B2 galaxies. In more recent works Parma et al. (1993) and Morganti et al. (1997) have analysed the presence of jets on the B2 galaxies using a whole set of VLA observations at various resolutions, including new unpublished data. From this analysis eight ill-defined jets have been now confirmed, another one was rejected (B2 1609+31) and three new radio jet sources were identified (B2 1615+32, B2 1833+32 and B2 $0828+32)$.

The list of B2 galaxies studied in this work is presented in Table 1. Information is given on the names of the optical galaxy/radio source in other catalogues, position of the optical galaxy, radio power, redshift, presence of radio jets (from Parma et al. 1987, 1993 and Morganti et al. 1997) and references for radio maps. Positions of the optical galaxies were taken from the original papers by $\mathrm{C} 75$ and F78, except for a few cases. For B2 1300+32 and B2 $1422+26$ we used the positions given by F87 and for B2 $1141+37$ that given by Giovannini et al. (1988). In two additional cases the positions were obtained from the CCD images, using as reference APM positions from POSS-I prints (Irwin 1992). These sources are B2 0838+32, whose position in $\mathrm{C} 75$ corresponds to the mid point between two galaxy components and B2 $1610+29$, whose position in the same work has a rather large offset.
For a few B2 sources the original identification papers by $\mathrm{C} 75$ and F78 listed more than one optical galaxy that could be associated to the radio emission. In the series of papers on VLA observations by the Bologna group (F87 and references therein) the optical counterparts were identified more precisely as the resolution of the radio maps increased and from the detection of the radio cores. The optical galaxy listed in Table 1 for B2 1141+37 (one of the galaxies in a group of four) is different from that given by F87. In addition, there are four sources in Table 1 for which one of the tentative identifications given by the Bologna group is selected in our work (B2 0838+32, B2 $1106+26$, B2 $1502+26$ and B2 1511+26). The explanation of the selection of the optical galaxy in these cases is presented in next section. For the source B2 $0843+31$ we listed the same optical galaxy as that given by the Bologna group, although the identification is uncertain. The ambiguity in the identification was previously noted by de Ruiter et al. (1986) and F87. The identification of source B2 0916+33 with a bright galaxy appears to be erroneous. C75 identified the source with an irregular galaxy, and the source was not further studied by the Bologna group since their follow up radio observations were restricted to the elliptical galaxies. A bright spiral galaxy was identified with B2 $1441+26$ in C75, but this appears to be another 
Table 2. Flux levels on the $V$-band contour plots

\begin{tabular}{|c|c|c|c|c|c|}
\hline B2 name & $\mu_{\mathrm{sky}}^{a}$ & $\mu_{\text {first }}$ & $\Delta$ mag & $\sigma$ level & seeing $(\operatorname{arcsec})$ \\
\hline $0116+31$ & 20.77 & 24.33 & 0.60 & 0.75 & 1.72 \\
\hline $0120+33$ & 20.71 & 24.92 & 0.60 & 1.00 & 1.85 \\
\hline $0149+35$ & 21.01 & 24.11 & 0.60 & 2.50 & 2.00 \\
\hline $0207+38$ & 21.00 & 23.88 & 0.60 & 3.00 & 1.96 \\
\hline $0209+37$ & 21.27 & 24.47 & 0.60 & 2.50 & 1.65 \\
\hline $0222+36$ & 21.05 & 24.13 & 0.60 & 2.50 & 1.94 \\
\hline $0258+35$ & 20.97 & 23.99 & 0.60 & 0.75 & 1.85 \\
\hline $0326+39$ & 20.95 & 25.06 & 0.60 & 1.00 & 1.69 \\
\hline $0331+39$ & 21.06 & 23.77 & 0.60 & 2.50 & 1.95 \\
\hline $0648+27$ & 20.77 & 23.95 & 0.60 & 1.00 & 2.16 \\
\hline $0708+32$ & 20.87 & 24.57 & 0.60 & 1.50 & 1.23 \\
\hline $0722+30$ & 20.89 & 24.24 & 0.60 & 2.00 & 2.21 \\
\hline $0800+24$ & 20.88 & 24.12 & 0.60 & 1.50 & 2.10 \\
\hline $0828+32$ & 21.04 & 23.81 & 0.60 & 1.00 & 1.45 \\
\hline $0838+32$ & 20.99 & 24.36 & 0.60 & 0.50 & 1.29 \\
\hline $0843+31$ & 20.86 & 24.56 & 0.60 & 1.50 & 1.89 \\
\hline $0916+33$ & 20.84 & 24.18 & 0.60 & 0.50 & 1.76 \\
\hline $0922+36$ & 21.01 & 24.08 & 0.60 & 2.50 & 1.12 \\
\hline $0924+30$ & 21.26 & 24.86 & 0.60 & 1.50 & 1.86 \\
\hline $0944+39$ & 20.29 & 24.38 & 0.60 & 1.00 & 2.35 \\
\hline $1003+26$ & 20.96 & 24.17 & 0.60 & 0.75 & 1.49 \\
\hline $1003+35$ & 21.01 & 24.64 & 0.60 & 1.50 & 1.10 \\
\hline $1037+30$ & 20.82 & 24.02 & 0.60 & 1.00 & 1.62 \\
\hline $1039+27$ & 21.16 & 24.50 & 0.60 & 2.00 & 2.14 \\
\hline $1101+38$ & 20.73 & 21.90 & 0.60 & 8.00 & 1.52 \\
\hline $1102+30$ & 20.99 & 24.32 & 0.60 & 0.50 & 1.13 \\
\hline $1106+26$ & 20.87 & 24.25 & 0.60 & 1.50 & 0.95 \\
\hline $1113+24$ & 20.93 & 24.62 & 0.60 & 1.50 & 1.63 \\
\hline $1141+37$ & 21.01 & 24.67 & 0.60 & 1.50 & 1.76 \\
\hline $1144+35$ & 20.80 & 24.21 & 0.60 & 2.00 & 1.38 \\
\hline $1204+24$ & 20.94 & 24.60 & 0.60 & 1.50 & 1.36 \\
\hline $1204+34$ & 21.15 & 24.59 & 0.60 & 1.50 & 2.56 \\
\hline $1217+29$ & 18.97 & 22.08 & 0.60 & 2.50 & 1.36 \\
\hline $1251+27$ & 21.05 & 24.34 & 0.60 & 1.50 & 0.99 \\
\hline $1256+28$ & 21.14 & 23.46 & 0.60 & 2.00 & 2.44 \\
\hline $1257+28$ & 21.14 & 23.94 & 0.60 & 2.50 & 1.34 \\
\hline $1300+32$ & 20.99 & 24.22 & 0.60 & 0.50 & 1.33 \\
\hline $1303+31$ & 21.02 & 24.33 & 0.60 & 2.00 & 1.44 \\
\hline $1316+29$ & 21.21 & 24.14 & 0.60 & 2.00 & 1.46 \\
\hline $1317+33$ & 21.07 & 23.51 & 0.60 & 1.50 & 1.64 \\
\hline $1318+34$ & 21.20 & 24.09 & 0.60 & 2.00 & 1.38 \\
\hline $1339+26$ & 21.09 & 23.78 & 0.60 & 2.00 & 1.70 \\
\hline $1346+26$ & 21.19 & 24.13 & 0.60 & 2.00 & 1.92 \\
\hline $1347+28$ & 21.41 & 24.23 & 0.60 & 1.00 & 1.27 \\
\hline $1358+30$ & 21.18 & 24.41 & 0.60 & 1.00 & 1.91 \\
\hline $1422+26$ & 21.41 & 24.27 & 0.60 & 2.00 & 1.26 \\
\hline $1430+25$ & 21.21 & 24.14 & 0.60 & 2.00 & 1.18 \\
\hline $1441+26$ & 21.10 & 24.04 & 0.60 & 2.00 & 1.68 \\
\hline $1447+27$ & 21.18 & 23.33 & 0.60 & 2.00 & 1.28 \\
\hline $1455+28$ & 21.42 & 24.27 & 0.75 & 2.00 & 1.26 \\
\hline $1457+29$ & 21.14 & 24.06 & 0.75 & 2.00 & 2.02 \\
\hline $1502+26$ & 21.03 & 23.32 & 0.60 & 1.50 & 1.02 \\
\hline $1511+26$ & 21.10 & 24.35 & 0.75 & 1.50 & 1.83 \\
\hline $1512+30$ & 21.43 & 24.59 & 0.60 & 1.50 & 1.31 \\
\hline $1527+30$ & 21.14 & 24.10 & 0.60 & 2.00 & 1.49 \\
\hline $1557+26$ & 21.09 & 24.34 & 0.60 & 0.50 & 1.45 \\
\hline $1609+31$ & 20.90 & 24.25 & 0.60 & 1.50 & 1.75 \\
\hline $1610+29$ & 21.14 & 24.10 & 0.60 & 2.00 & 1.36 \\
\hline
\end{tabular}

Table 2. continued

\begin{tabular}{cccccc} 
B2 name & $\mu_{\text {sky }}^{a}$ & $\mu_{\text {first }}$ & $\Delta$ mag & $\sigma$ level & seeing(arcsec) \\
\hline $1615+32$ & 21.07 & 23.74 & 0.75 & 2.00 & 2.08 \\
$1615+35$ & 20.92 & 24.26 & 0.60 & 1.50 & 1.31 \\
$1621+38$ & 21.09 & 23.86 & 0.60 & 1.00 & 1.05 \\
$1626+39$ & 21.08 & 23.92 & 0.60 & 1.00 & 1.29 \\
$1652+39$ & 21.07 & 23.17 & 0.60 & 8.00 & 1.03 \\
$1657+32$ & 21.18 & 24.14 & 0.60 & 1.00 & 1.37 \\
$1658+32$ & 20.97 & 24.02 & 0.60 & 2.00 & 1.46 \\
$1726+31$ & 21.02 & 24.33 & 0.75 & 1.50 & 1.13 \\
$1833+32$ & 20.92 & 23.99 & 0.60 & 2.00 & 1.53 \\
$1855+37$ & 20.91 & 23.53 & 0.60 & 2.00 & 1.23 \\
$2240+29$ & 20.79 & 23.46 & 0.60 & 3.00 & 1.25 \\
$2257+25$ & 19.20 & 22.67 & 0.60 & 0.50 & 1.26 \\
$2320+32$ & 19.59 & 21.98 & 0.60 & 1.00 & 1.79 \\
$2335+30$ & 20.86 & 24.48 & 0.60 & 0.50 & 2.03 \\
\hline
\end{tabular}

${ }^{a} V \operatorname{mag} \operatorname{arcsec}^{-2}$.

misidentification (Parma, private communication). A description of the optical identification of these three radio sources is presented in the next section.

The redshifts listed in Table 1 were taken from $\mathrm{C} 75$ and F78, except for B2 1457+29, which was obtained in this work, and B2 1039+27, B2 1106+26, B2 1141+37, B2 $1300+32$, B2 1317+33, B2 1339+26 and B2 1502+26, whose redshifts were taken from the NASA/IPAC Extragalactic Database (NED) ${ }^{1}$.

The total radio power at $408 \mathrm{MHz}$ was calculated using the total flux densities at $408 \mathrm{MHz}$ given by C75, F78 and the revised values (for some sources) presented by Feretti et al. (1984) and Giovannini et al. (1988). An spectral index $\alpha=-0.7\left(S_{\nu} \propto \nu^{\alpha}\right)$ was assumed to obtain the $k$ correction. The used expression was $\log P_{408}=24.20+\log$ $S_{408}+2 \log (z(1+z / 2))-\log (1+z)^{1+\alpha}$, being $S_{408}$ the total flux density at $408 \mathrm{MHz}$ in mJy, $z$ the redshift and $P_{408}$ the total radio power at $408 \mathrm{MHz}$ in $\mathrm{W} \mathrm{Hz}^{-1}$.

\section{Observations and data reduction}

The $V$-band CCD images were obtained in two runs, on 1994 December 30-31, 1995 January 1-2, and 1995 May 26-29, at the $1 \mathrm{~m}$ Jacobus Kapteyn Telescope at the Observatorio del Roque de los Muchachos (La Palma, Canary Islands). The chip used was a TEK with $1024 \times$ 1024 pixels, providing a field of view at the $\mathrm{f} / 15$ focus of the JKT of $5.6 \times 5.6 \mathrm{arcmin}^{2}$. Typically two $900 \mathrm{~s}$ exposures were taken for each object in order to diminish the effects of cosmic-ray hits, saturation or guiding problems. Each couple of frames was then coadded after data reduction. Typical seeing was in the range $1-1.5$ arcsec.

\footnotetext{
1 The NASA/IPAC Extragalactic database (NED) is operated by the Jet Propulsion Laboratory, California Institute of Technology, under contract with the National Aeronautics and Space Administration.
} 
Photometric standard stars were observed all nights in order to flux calibrate the images. The photometry zeropoint errors were in the range $0.02-0.1$ being in average of $\sim 0.05$ mag. Atmospheric extinction coefficients were around 0.15 mag airmass ${ }^{-1}$.

During some of the nights we obtained short exposures of pairs of bright stars from the PPM catalogue (Roeser \& Bastian 1988) in order to measure the pixel scale and orientation of the chip. The resulting scale of the frames derived from 3 pairs of stars was $0.3301 \pm 0.0003 \mathrm{arcsec} / \mathrm{pixel}$ and the position of the North was $0.8( \pm 0.01)$ clockwise from the frame $Y$ axis. The preliminary reduction (bias subtraction, flat-field correction) was done using standard routines from the IRAF $^{2}$ package. No special processing was done for the flat-fielding and we used sky flats. Flatfield correction was better than $0.7 \%$.

The sky background of the images was calculated using two methods. The first was the simple technique in which the mean sky level is measured in regions free of interfering objects, evenly distributed accross the images. A second method consisted on computing the histogram of intensities on the frames and, after applying a clipping algorithm, estimate the mean value from the histogram. The sky background estimations obtained with the two methods typically agreed within $\sim 0.5 \%$. For the cases where a bright star produced a large extended emission, this emission was modelled and substracted from the original frame.

B2 $1457+29$ was observed during a service night at the WHT $4.2 \mathrm{~m}$ telescope using the ISIS double spectrograph to measure its redshift. A slit width of 2 arcsec was used and the dispersion was of $121 \AA / \mathrm{mm}$ providing a spectral resolution of $\sim 7 \AA$. Standard procedures to calibrate the spectra were carried out. The absortion lines $\mathrm{NaD}, \mathrm{MgB}$, Fe 1 and $\mathrm{Ca} 1$ were used and we obtained a redshift of $z=0.146 \pm 0.003$.

Figure 1 shows $V$-band contour maps of the galaxies. The galaxies are identified in these maps, except for the obvious cases. The images were median-filtered before the contour maps were obtained. The sky brightness $\left(\mu_{\text {sky }}\right)$, the first contour $\left(\mu_{\text {first }}\right)$, the contour interval $(\Delta \mathrm{mag})$, the signal-to-noise ratio for the first contour ( $\sigma$ level), and the seeing are indicated for each map in Table 2. The magnitudes have been corrected for Galactic extinction (Burstein \& Heiles 1982). On each figure we give the scale in $\mathrm{kpc}$ for the adopted cosmology.

For five of the sources in Table 1 the identified optical galaxy is different from that given in the series of papers by the Bologna group (C75, F78, F87 and references therein) or we have selected one of the tentative identifications given in these papers. In addition, for three

\footnotetext{
${ }^{2}$ IRAF is distributed by the National Optical Astronomy Observatories, which is operated by Association of Universities for Research in Astronomy, Inc., under cooperative agreement with the National Science Foundation.
}

radio sources in Table 1 the association with the optical galaxies is uncertain or wrong. The identification for these eight cases is described below.

B2 0838+32. The optical counterpart given by F87 consists of a galaxy and a fainter companion at 11 arcsec SE (see Fig. 1). The brighter galaxy has a better spatial coincidence with the radio emisison in the map presented at F86, and we therefore quote the position of this galaxy. The optical position listed by F87 was slightly shifted towards the fainter galaxy.

B2 0843+31. de Ruiter et al. (1986) and F87 note that the identification with the optical galaxy is uncertain, since the VLA radio map shows a bright unresolved component towards the south, which could be related to a background object. The CCD image does not show an optical counterpart of this source down to a limiting magnitude $V \sim 23-23.5 \mathrm{mag}$.

B2 $0916+33$. We noted a large offset between the position of the irregular galaxy and that quoted by $\mathrm{C} 75$. There are no published radio maps of this source, but its position is included in the VLA FIRST Survey (White et al. 1997). The FIRST image shows a faint double source coincident with the position of the optical galaxy. The optical galaxy has a double nucleus, each of them coincident with one of the radio emission peaks. 43 arcsec SW of the galaxy there is a brighter pointlike radio source (R.A.(1950) $=$ $09^{\mathrm{h}} 16^{\mathrm{m}} 49.2$ and Dec. $\left.(1950)=+33^{\circ} 07^{\prime} 54^{\prime \prime}\right)$, coincident with a bright pointlike object (R.A.(1950) $=09^{\mathrm{h}} 16^{\mathrm{m}} 48^{\mathrm{s}} .7$ and Dec. $\left.(1950)=+33^{\circ} 07^{\prime} 54^{\prime \prime}\right)$, with $V=18.45$ mag on our image. The better similarity of this position with that given by $\mathrm{C} 75$ and the agreement in radio flux indicates that the pointlike optical object is likely the counterpart of the B2 source. In fact C75 quote $S_{1415}=100 \mathrm{mJy}$ for the B2 source and FIRST and NRAO/VLA Sky Survey (NVSS, Condon et al. 1998) quote respectively 89 and $93 \mathrm{mJy}$ for the SW radio source and $22 \mathrm{mJy}$ (similar value for both surveys) for the irregular galaxy. The new identification is indicated with an arrow in Fig. 1.

B2 1106+26. C75 list two optical galaxies for this radio source. The northern galaxy is the elliptical NGC 3534 and shows a better agreement with the radio position of the source in Douglas et al. (1996, Texas Survey).

B2 1141+37. The optical counterpart given at F87 is the most sourthern one of a tight chain of four galaxies. We selected the galaxy marked in Fig. 1 as counterpart because it shows a better spatial coincidence with the central emission on the radio map presented by de Ruiter et al. (1986). This is also the optical counterpart given by Giovannini et al. (1988), who detected the core at $5 \mathrm{GHz}$ at the position of this galaxy.

B2 1441+26. This source was identified with a spiral galaxy located at the center of the two lobes of the radio source. The $20 \mathrm{~cm}$ FIRST data and a $6 \mathrm{~cm}$ unpublished map (Parma, private communication) show that the core of the radio source is embedded in the eastern lobe and is located $\sim 36$ arcsec East of the spiral galaxy. 
Table 3. Visual magnitudes of the B2 galaxies

\begin{tabular}{|c|c|c|c|c|c|c|}
\hline B2 name & $m_{V}$ & $m_{V}-A_{V}$ & Aperture $^{a}$ & $M_{V}$ & Type & Morph features \\
\hline $0116+31$ & 14.47 & 14.37 & 33.0 & -22.55 & $\mathrm{E}$ & dumbbell, dist \\
\hline $0120+33$ & 11.43 & 11.33 & 128.7 & -22.77 & $\mathrm{E}$ & dist, $\mathrm{BCM}$ (or 2nd) $\mathrm{Zw}_{\mathrm{w}} 0107.5+3212$ \\
\hline $0149+35$ & 12.40 & 12.26 & 66.0 & -21.77 & $\mathrm{E}$ & dust, BCM A262 \\
\hline $0207+38$ & 12.57 & 12.39 & 49.5 & -21.91 & Irr & dust \\
\hline $0209+37$ & 15.61 & 15.45 & 17.2 & -18.81 & Irr & \\
\hline $0222+36$ & 13.93 & 13.76 & 26.4 & -21.84 & $\mathrm{E}$ & tail \\
\hline $0258+35$ & 12.26 & 12.07 & 69.3 & -21.96 & $\mathrm{E}$ & shell \\
\hline $0326+39$ & 13.27 & 12.95 & 39.6 & -22.01 & $\mathrm{E}$ & \\
\hline $0331+39$ & 12.99 & 12.66 & 45.2 & -21.89 & $\mathrm{E}$ & \\
\hline $0648+27$ & 13.82 & 13.45 & 36.6 & -22.65 & $\mathrm{E}$ & dist \\
\hline $0708+32$ & 15.51 & 15.29 & 23.8 & -21.92 & $\mathrm{E}$ & \\
\hline $0722+30$ & 15.04 & 14.85 & 21.5 & -19.58 & $\mathrm{~S}(\mathrm{~S} 0)$ & \\
\hline $0800+24$ & 14.96 & 14.83 & 28.1 & -21.40 & $\mathrm{E}$ & \\
\hline $0828+32$ & 15.03 & 14.95 & 23.1 & -21.63 & $\mathrm{E}$ & \\
\hline $0838+32$ & 14.48 & 14.41 & 33.0 & -22.82 & $\mathrm{E}$ & mult nucl \\
\hline $0843+31$ & 16.36 & 16.30 & 23.8 & -20.89 & $\mathrm{E}$ & \\
\hline $0922+36$ & 15.43 & 15.39 & 26.4 & -22.98 & $\mathrm{E}$ & \\
\hline $0924+30$ & 13.32 & 13.28 & 41.9 & -21.87 & $\mathrm{E}$ & dust? \\
\hline $0944+39$ & 14.68 & 14.68 & 22.8 & -21.43 & $\mathrm{~S}$ & \\
\hline $1003+26$ & 15.47 & 15.47 & 19.8 & -22.98 & $\mathrm{E}$ & BCM A923 \\
\hline $1003+35$ & 15.71 & 15.71 & 25.7 & -22.37 & $\mathrm{E}$ & \\
\hline $1037+30$ & 15.81 & 15.81 & 19.8 & -22.08 & $\mathrm{E} ?$ & dist E/Irr \\
\hline $1039+27$ & 14.48 & 14.48 & 31.4 & -20.08 & $\mathrm{~S}$ & \\
\hline $1101+38$ & - & - & - & - & $\mathrm{E}$ & nucleus \\
\hline $1102+30$ & 14.57 & 14.57 & 32.7 & -22.79 & $\mathrm{E}$ & \\
\hline $1106+26$ & 13.94 & 13.94 & 39.6 & -20.79 & $\mathrm{~S}$ & \\
\hline $1113+24$ & 15.10 & 15.10 & 14.9 & -23.05 & $\mathrm{E}$ & BCM Zw 1113.0+2452 \\
\hline $1141+37$ & - & - & - & - & $\mathrm{E}$ & chain of galaxies \\
\hline $1144+35$ & 14.60 & 14.60 & 33.7 & -22.46 & $\mathrm{E}$ & \\
\hline $1204+24$ & 15.35 & 15.35 & 31.4 & -22.16 & $\mathrm{E}$ & \\
\hline $1204+34$ & 15.62 & 15.62 & 23.1 & -21.95 & $\mathrm{E}$ & \\
\hline $1217+29$ & 10.41 & 10.41 & 89.1 & -19.20 & $\mathrm{E}$ & \\
\hline $1251+27$ & 15.96 & 15.96 & 16.5 & -21.80 & $\mathrm{E}$ & \\
\hline $1256+28$ & 13.88 & 13.88 & 25.1 & -20.90 & $\mathrm{E}$ & Coma \\
\hline $1257+28$ & 11.94 & 11.94 & 85.8 & -22.98 & $\mathrm{E}$ & 2nd BCM Coma \\
\hline $1300+32$ & 16.76 & 16.76 & 14.2 & -22.21 & $\mathrm{E}$ & \\
\hline $1303+31$ & 16.59 & 16.59 & 12.2 & -22.89 & Irr & BCM A1667 \\
\hline $1316+29$ & 15.39 & 15.39 & 21.5 & -22.00 & $\mathrm{E}$ & \\
\hline $1317+33$ & 14.25 & 14.25 & 19.8 & -21.59 & $\mathrm{E}$ & dumbbell \\
\hline $1318+34$ & 13.77 & 13.77 & 54.5 & -21.08 & Irr & \\
\hline $1339+26$ & 15.10 & 15.10 & 11.6 & -22.36 & $\mathrm{E}$ & dumbbell \\
\hline $1346+26$ & 14.11 & 14.11 & 33.0 & -22.96 & $\mathrm{E}$ & BCM A1795 \\
\hline $1347+28$ & 15.66 & 15.66 & 19.8 & -21.72 & $\mathrm{E}$ & \\
\hline $1358+30$ & 16.79 & 16.79 & 11.6 & -21.54 & $\mathrm{E} ?$ & \\
\hline $1422+26$ & 14.32 & 14.32 & 31.4 & -21.56 & $\mathrm{E}$ & \\
\hline $1430+25$ & 16.65 & 16.65 & 13.2 & -20.99 & $\mathrm{E}$ & \\
\hline $1447+27$ & 13.88 & 13.88 & 33.0 & -21.58 & $\mathrm{E}$ & \\
\hline $1455+28$ & 16.89 & 16.89 & 14.9 & -22.01 & $\mathrm{E}$ & \\
\hline $1457+29$ & 16.96 & 16.97 & 13.2 & -22.03 & $\mathrm{E}$ & \\
\hline $1502+26$ & 15.42 & 15.42 & 11.6 & -21.28 & $\mathrm{E}$ & dumbbell \\
\hline $1511+26$ & 17.68 & 17.68 & 3.3 & -20.59 & $\mathrm{E}$ & dumbbell \\
\hline $1512+30$ & 15.37 & 15.37 & 19.8 & -22.57 & $\mathrm{E}$ & \\
\hline
\end{tabular}


Table 3. continued

\begin{tabular}{lccrrll} 
B2 name & $m_{V}$ & $m_{V}-A_{V}$ & Aperture $^{a}$ & $M_{V}$ & Type & Morph features \\
\hline $1527+30$ & 15.64 & 15.64 & 15.5 & -22.77 & $\mathrm{E}$ & \\
$1557+26$ & 14.97 & 14.94 & 16.5 & -21.33 & $\mathrm{E}$ & \\
$1609+31$ & 16.21 & 16.18 & 18.2 & -21.79 & $\mathrm{E}$ & \\
$1610+29$ & 13.29 & 13.26 & 31.4 & -22.25 & $\mathrm{E}$ & BCM A2162 \\
$1615+32$ & 15.79 & 15.76 & 21.5 & -23.31 & $\mathrm{Irr}$ & \\
$1615+35$ & 13.54 & 13.51 & 34.7 & -21.88 & $\mathrm{E}$ & \\
$1621+38$ & 12.68 & 12.65 & 54.5 & -22.84 & $\mathrm{E}$ & \\
$1626+39$ & 12.42 & 12.39 & 57.8 & -23.05 & $\mathrm{E}$ & mult nucl, BCM A2199 \\
$1652+39$ & 12.89 & 12.85 & 42.9 & -22.83 & $\mathrm{E}$ & nucleus \\
$1657+32$ & 15.40 & 15.35 & 23.1 & -21.72 & $\mathrm{E}$ & \\
$1658+32$ & 16.11 & 16.06 & 18.2 & -22.09 & $\mathrm{E}$ & \\
$1726+31$ & 17.04 & 16.97 & 8.9 & -22.32 & $\mathrm{E}$ & \\
$1833+32$ & 14.00 & 13.83 & 15.2 & -23.08 & $\mathrm{E}$ & nucleus, dist \\
$1855+37$ & 14.05 & 13.84 & 19.8 & -22.93 & $\mathrm{E}$ & \\
$2240+29$ & 13.98 & 13.88 & 19.8 & -21.17 & $\mathrm{~S}$ & nucleus \\
$2257+25$ & 15.22 & 15.15 & 18.2 & -19.80 & $\mathrm{~S}$ & \\
$2320+32$ & 14.29 & 14.20 & 16.5 & -20.06 & Irr & shell \\
$2335+30$ & 14.35 & 14.25 & 49.5 & -13.27 & Irr & \\
\hline
\end{tabular}

${ }^{a}$ Aperture radius in arcsecs.

NOTE. The absolute $V$ magnitudes were obtained using the expression: $M_{V}=m_{V}-A_{V}-43.00-5 \log$ $(z(1+z / 2))$. The photometry of the galaxies B2 $1101+38$ and $1141+37$ is not presented. For the first case the nucleus of the image is saturated in the two images obtained. The second source was excluded since its emission was strongly contaminated by companion galaxies.

The core is spatially coincident with a point-like object with $V=18.88 \mathrm{mag}$ and coordinates R.A.(1950) = $14^{\mathrm{h}} 41^{\mathrm{m}} 56^{\mathrm{s}} .3$ and Dec. $(1950)=26^{\circ} 14^{\prime} 06^{\prime \prime} \cdot 1$. This source is indicated with an arrow in the contour map in Fig. 1.

B2 1502+26, 3C 310. This source was originally identified as a galaxy pair in F78. The high resolution radio map obtained by van Breugel \& Jägers (1982) shows that the western optical galaxy has a better spatial coincidence with the radio core. Smith \& Heckman (1989a) also identify the western galaxy as optical counterpart.

B2 1511+26, 3C 315. This source was originally identified as a galaxy pair in F78 and F87. The northern galaxy shows a better positional coincidence with the radio core in the high resolution radio map by F87 and we therefore select this source as optical counterpart. The same identification is given by Smith \& Heckman (1989a).

\section{4. $V$-band magnitudes and morphology}

The $V$ magnitudes of the galaxies were measured using circular apertures with the radius at which the intensity gradient reached zero. Stars, multiple nuclei or other galaxies within the outline of the radio galaxy were removed, sustituting their counts by the mean intensity value in adjacent positions. The apparent $V$ magnitudes, aperture radius, $V$ magnitudes corrected for Galactic extinction and absolute magnitudes (without $k$ and evolutionary corrections) are listed in Table 3. The main source of error in the apparent magnitudes is the zero-point photometric calibration, which is, in average, 0.05 mag. The classification of the galaxies as ellipticals (E), spirals (S) or irregulars (Irr), obtained from the contour plots, is also given in this table. In total 57 galaxies correspond to ellipticals, 6 to spirals (including a possible SO) and 7 to irregulars. Along with this classification we listed some additional remarks on the optical morphology of the ellipticals, like isophote distortions relative to an elliptical model, shells, tails, nuclear dust, a strong nucleus, or multiple nuclei. Some of these peculiarities (e.g. distorted morphology and presence of tails, shells or nuclear dust) are generally interpreted as signatures of gravitational interaction (Schweizer 1998). In Table 3 we also give information about the association with known cluster of galaxies (Abell or Zwicky clusters) or with dumbbell systems. Ten radio sources (14\%) are located in clusters of galaxies of which 8 are the brightest cluster members (BCM) and one is the second ranked galaxy in the cluster (2nd BCM). Five radio sources (7\%) belong to dumbbell systems and 2 appear to have multiple nuclei. A detailed and quantitative study of the environment of the complete B2 sample will be presented in a forthcoming paper.

\section{Surface photometry}

\subsection{Procedure and results}

Surface photometry of the observed galaxies was performed using the method described by González-Serrano 


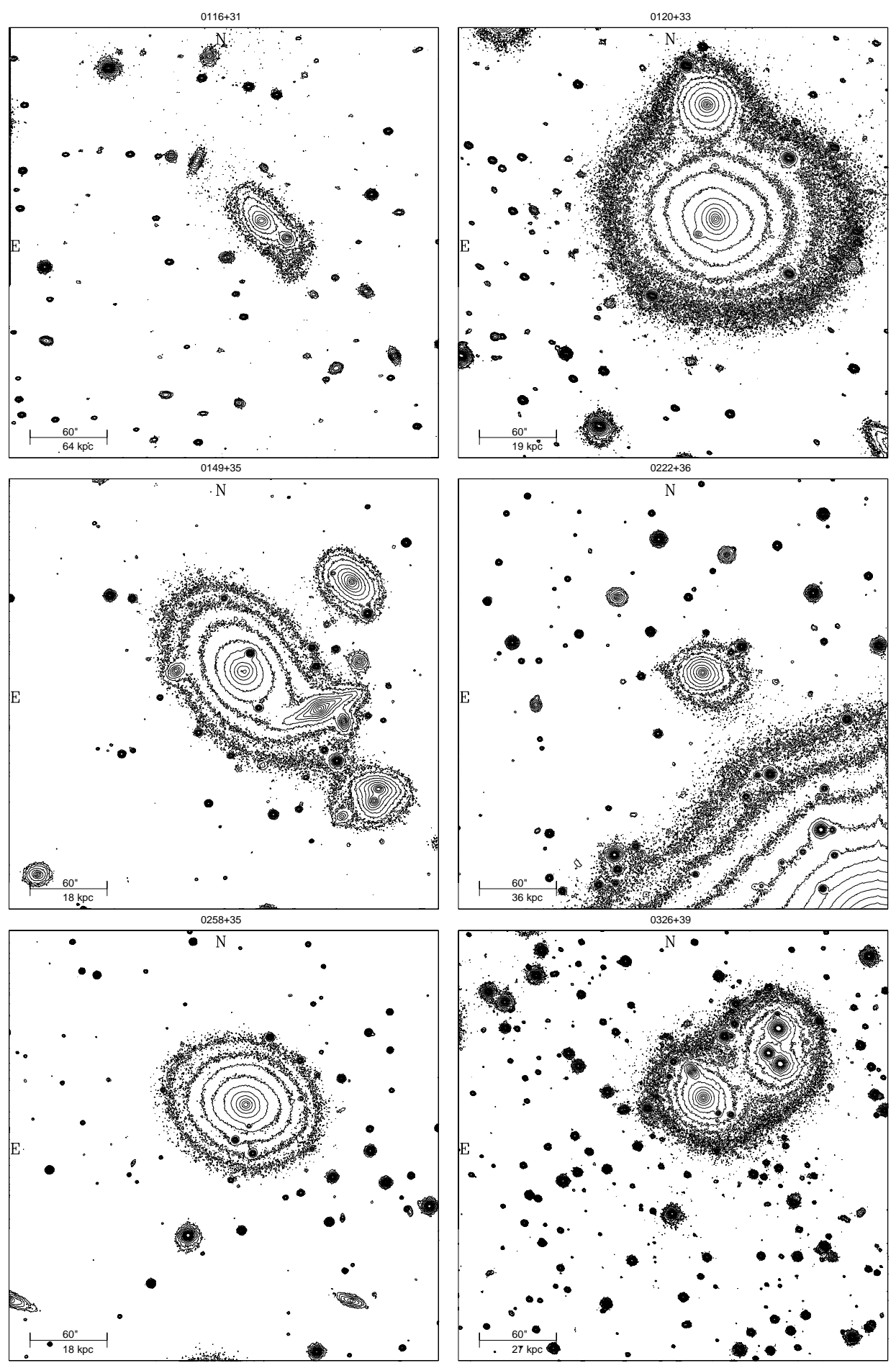

Fig. 1. $V$-band contour maps of the observed B2 galaxies. Orientation is the standard (North up and East to the left) although the North is exactly 0.8 clockwise from the vertical axis. The contour levels, corrected from Galactic reddening, are indicated in Table 2 
\& Pérez-Fournon (1991) and González-Serrano et al. (1993). The procedure consists of a Fourier analysis of the intensity variations along elliptical contours fitted to the galaxy intensity distribution. For a given semimajor axis the following ellipse parameters were obtained: centre coordinates, ellipticity, position angle of the major axis, mean intensity and the first four Fourier components. Of these, the third $(\cos 3 \theta)$ and fourth $(\cos 4 \theta)$ give information of non-elliptical isophotes and their distortions (see González-Serrano \& Pérez-Fournon 1991 and references therein). After an ellipse was fitted, the semimajor axis was increased by a fixed factor, proceeding to the next ellipse. In this way radial profiles were obtained for all the ellipse parameters, including the surface brightness profile. In most cases the surface photometry analysis was started at the first pixel from the nucleus of the galaxy and finished when the ellipses reached the sky background level. Some galaxies were saturated in their nuclear parts and the analysis was started at larger radii from the centre.

Before the method was applied each galaxy image was cleaned from other objects, bad pixels and cosmic ray hits that could contaminate the intensity distribution of the galaxy. This was done automatically by building a mask image that indicates the pixels to be ignored in the ellipse fitting process. When the galaxy under study had a nearby companion the cleaning task was more complicated since the companion, if bright and closeby, contributed to the light distribution of the main galaxy over a large area. In these cases we have applied an iterative procedure in order to subtract the contaminating galaxy from the main image. This technique is explained in detail in GonzálezSerrano \& Pérez-Fournon (1991), where a discussion on the reliability and errors is also given. Typical errors of ellipse parameters are below $\sim 3^{\circ}$ for $\mathrm{PA}$, below $\sim 0.02$ for ellipticity, and $\sim 0.5 \%$ for the $B_{4}$ parameter. Deviations lower than these errors were found from the comparison of the PA and ellipticity profiles of B2 $1217+29$ (NGC 4278) and B2 1610+29 (NGC 6086) with those presented in Peletier et al. (1990).

The resulting surface brightness profiles (expressed in $V$ magnitudes corrected for Galactic extinction) and isophote parameters' profiles, including ellipticity, position angle and $B_{4}$, are presented in Fig. 2 and listed in Table 4. The profiles in the inner part of the galaxies are not reliable, as they are affected by the seeing. The seeing radius is indicated in each profile by a small vertical hash. The procedure of quantitative surface photometry was applied to 59 of the galaxies in the sample, including 53 ellipticals, 4 spirals and 2 irregulars. The galaxies excluded were 4 ellipticals (B2 1141+37, B2 1339+26, B2 1502+26 and B2 1511+26), 2 spirals (B2 $1106+26$ and B2 $2240+29$ ), and 5 irregulars (B2 0207+38, B2 0209+37, B2 1303+31, B2 $1318+34$ and B2 2320+32). The four ellipticals excluded belong to multiple systems (three dumbells and a tight chain of four galaxies) in which the secondary galax- ies seriously affected the light distribution of the main galaxy. The reason to exclude seven spirals/irregulars was that the presence of features like bars, spiral arms, dust fringes and/or double nuclei, produced a large deviation of the galaxies relative to an elliptical model.

\subsection{Surface brightness profiles of the elliptical galaxies}

The surface brightness profile of 45 of the 53 ellipticals reasonably fit an $r^{1 / 4}$ law. For one of these galaxies, B2 $0149+35$, the flat surface brightness profile at the central part is due to the presence of nuclear dust. Three galaxies show large excesses relative to the $r^{1 / 4}$ law in the outer parts (B2 0120+33, B2 0326+39, and B2 1447+27). These excesses are at least of one magnitude at the faintest isophote. The remaining 8 ellipticals are described below. For five of them the surface brightness profile is largely affected by the seeing due to a combination of a small galaxy size and a poor seeing. These galaxies are B2 $0843+31$, B2 $1204+34$, B2 $1358+30$, B2 $1455+28$ and B2 $1457+29$, and their surface brightness profiles $\left(\mu_{V}\right)$ appear severely curved on the $\mu_{V}-r^{1 / 4}$ plots (Fig. 2). We recall that the first of these sources is an uncertain identification. The three remaining ellipticals have bright nuclei which strongly affect the surface brightness profiles. These galaxies are B2 $1101+38$, B2 $1652+39$ and B2 $1833+32$, and their $\mu_{V}$ profiles clearly deviate from a de Vaucouleurs law. For these threee galaxies there is evidence of nuclear activity in the optical range from the literature. B2 $1101+38$ and B2 $1652+39$ are catalogued Markarian galaxies (Mrk 421 and Mrk 501 respectively), and hence have nuclei with blue/UV excess. Both are classified as BL Lac in Hewitt \& Burbidge (1993, see references for the sources therein). B2 $1833+32$ is a broad-line radio galaxy (Corbett et al. 1997 and references therein).

For the 45 galaxies obeying the $r^{1 / 4}$ law the surface brightness profiles were fitted with this law, and the effective radius $r_{\mathrm{e}}$ and the surface brightness at that radius $\mu_{\mathrm{e}}$ were obtained from the fits. Table 5 lists the values of both parameters, along with the ellipticity and position angle at the effective radius, and the minimum and maximum radii used for the $r^{1 / 4}$ fits. Columns $4-7$ list $\mu_{\mathrm{e}}$ in mag $\operatorname{arcsec}^{-2}$, corrected for Galactic extinction, $r_{\mathrm{e}}$ in $\operatorname{arcsecs}$ and their errors. Columns 10-12 list $\mu_{\mathrm{e}}$ in mag $\operatorname{arcsec}^{-2}$, including the $k$-correction from Coleman et al. (1980) and the $(1+z)^{-4}$ correction, and $r_{\mathrm{e}}$ and its error in kpc.

\subsection{Isophotal properties of the elliptical galaxies}

Five galaxies classified as ellipticals in Table 3 were noted to show significant distortions relative to an elliptical model from the contour maps in Fig. 1. These galaxies are B2 0116+31, B2 0120+33, B2 0648+27, B2 1037+37 and $\mathrm{B} 21833+32$. The distortion of $\mathrm{B} 21037+30$ is due to a 
Ellipticals

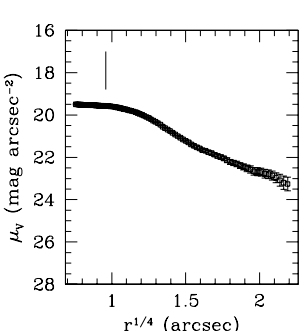

$0116+31$
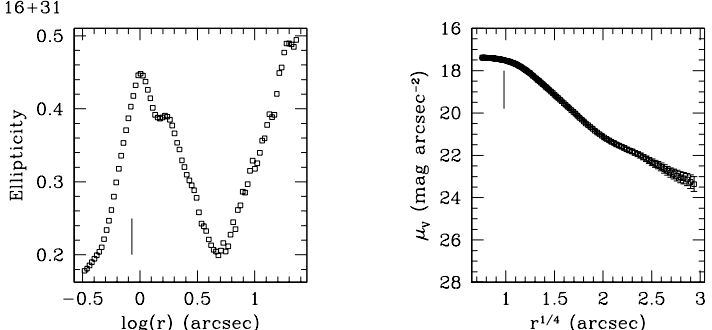

$0120+33$
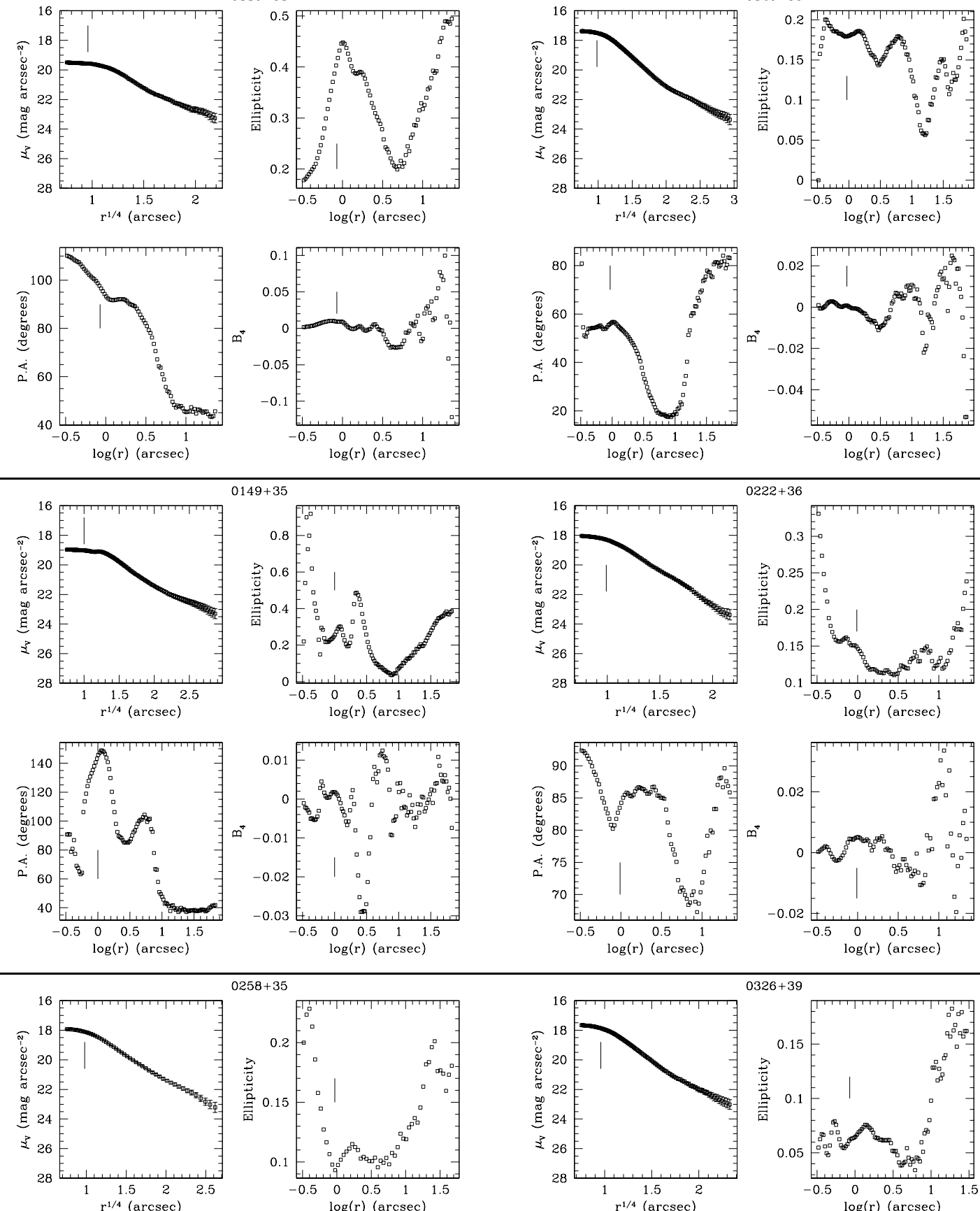

$0326+39$
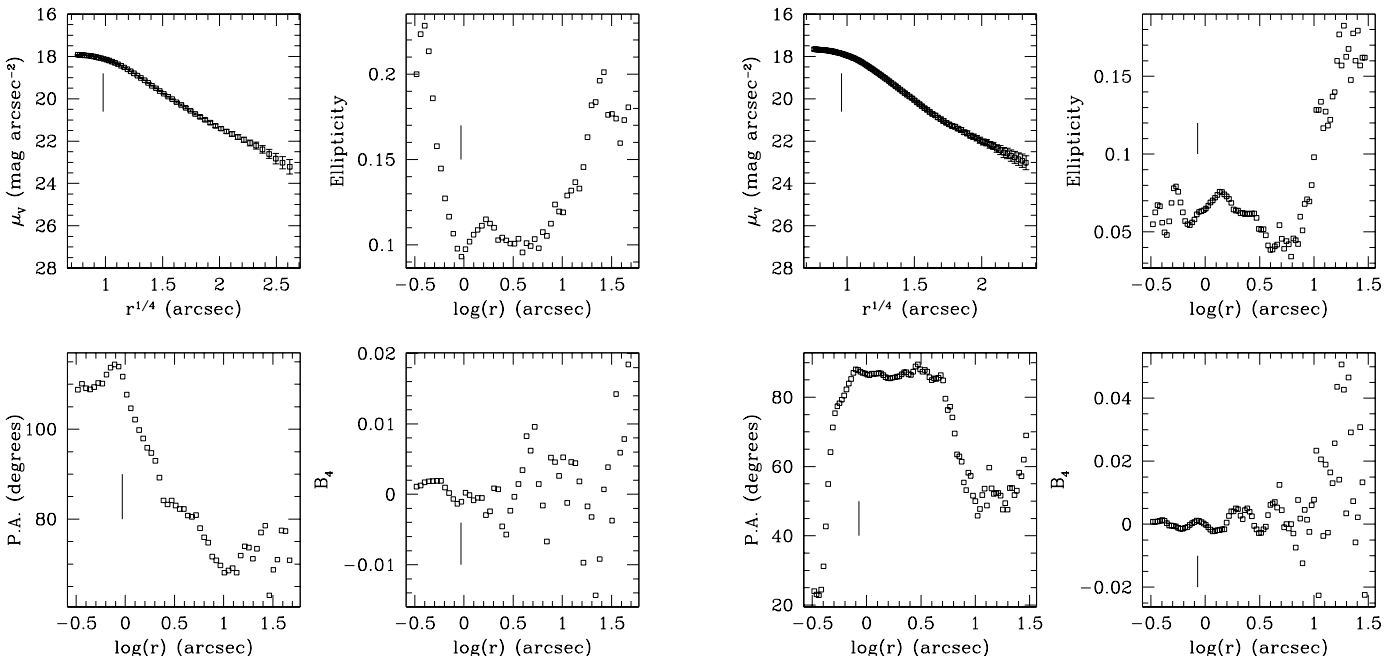

Fig. 2. Radial profiles for $V$-band surface brightness, ellipticity, position angle and $B_{4}$ parameter for the observed B2 galaxies. The seeing radius is indicated in each profile by a small vertical hash 
Table 5. Photometric and structural parameters of the $r^{1 / 4}$ galaxies

\begin{tabular}{|c|c|c|c|c|c|c|c|c|c|c|c|}
\hline B2 name & $r_{\min }$ & $r_{\max }$ & $\mu_{\mathrm{e}}$ & $\sigma\left(\mu_{\mathrm{e}}\right)$ & $r_{\mathrm{e}}\left({ }^{\prime \prime}\right)$ & $\sigma\left(r_{\mathrm{e}}\right)$ & $\epsilon$ & $\mathrm{PA}^{\circ}$ & $\mu_{\mathrm{e}}$ & $r_{\mathrm{e}}(\mathrm{kpc})$ & $\sigma\left(r_{\mathrm{e}}\right)$ \\
\hline $0116+31$ & 2.0 & 13.6 & 23.82 & 0.06 & 24.2 & 1.2 & 0.482 & 43.1 & 23.48 & 25.48 & 1.26 \\
\hline $0120+33$ & 2.0 & 21.4 & 21.76 & 0.02 & 23.1 & 0.4 & 0.121 & 66.1 & 21.66 & 7.16 & 0.12 \\
\hline $0149+35$ & 2.8 & 28.0 & 23.61 & 0.03 & 56.9 & 1.8 & 0.376 & 40.3 & 23.52 & 17.23 & 0.55 \\
\hline $0222+36$ & 2.0 & 21.4 & 21.93 & 0.05 & 11.6 & 0.4 & 0.123 & 77.8 & 21.74 & 7.01 & 0.24 \\
\hline $0258+35$ & 2.0 & 19.4 & 22.75 & 0.03 & 31.8 & 0.8 & 0.176 & 65.9 & 22.66 & 9.63 & 0.24 \\
\hline $0326+39$ & 2.0 & 10.5 & 21.28 & 0.21 & 9.5 & 1.1 & 0.008 & 52 & 21.14 & 4.32 & 0.50 \\
\hline $0331+29$ & 2.0 & 21.4 & 21.16 & 0.05 & 11.6 & 0.3 & 0.110 & 110.7 & 21.04 & 4.41 & 0.11 \\
\hline $0648+27$ & 2.0 & 19.5 & 21.07 & 0.08 & 9.0 & 0.4 & 0.389 & 39.8 & 20.83 & 6.72 & 0.30 \\
\hline $0708+32$ & 2.0 & 14.5 & 24.09 & 0.13 & 18.3 & 1.7 & 0.327 & 43.0 & 23.71 & 21.63 & 2.01 \\
\hline $0800+24$ & 2.0 & 14.5 & 22.54 & 0.07 & 10.2 & 0.5 & 0.311 & 48.9 & 22.29 & 8.03 & 0.39 \\
\hline $0828+32$ & 2.0 & 11.7 & 22.19 & 0.12 & 6.9 & 0.4 & 0.075 & 88.5 & 21.90 & 6.30 & 0.37 \\
\hline $0838+32$ & 2.0 & 18.7 & 23.83 & 0.07 & 23.2 & 1.3 & 0.266 & 130.8 & 23.44 & 27.71 & 1.55 \\
\hline $0922+36$ & 2.0 & 13.6 & 23.82 & 0.12 & 14.1 & 1.2 & 0.405 & 104.4 & 23.19 & 26.23 & 2.23 \\
\hline $0924+30$ & 2.4 & 34.9 & 23.20 & 0.05 & 28.4 & 1.1 & 0.149 & 46.4 & 23.05 & 14.08 & 0.55 \\
\hline $1003+26$ & 2.0 & 16.0 & 25.49 & 0.11 & 45.6 & 4.6 & 0.516 & 120.6 & 24.84 & 87.40 & 8.82 \\
\hline $1003+35$ & 2.0 & 10.0 & 22.89 & 0.16 & 7.1 & 0.7 & 0.310 & 43.8 & 22.33 & 11.83 & 1.17 \\
\hline $1037+30$ & 2.0 & 8.7 & 22.11 & 0.21 & 4.4 & 0.5 & 0.112 & 87.1 & 21.60 & 6.80 & 0.77 \\
\hline $1102+30$ & 2.0 & 18.0 & 22.74 & 0.09 & 10.5 & 0.6 & 0.118 & 141.7 & 22.33 & 13.21 & 0.75 \\
\hline $1113+24$ & 2.0 & 16.6 & 23.32 & 0.12 & 12.1 & 0.9 & 0.093 & 101.6 & 22.74 & 20.72 & 1.54 \\
\hline $1144+35$ & 2.0 & 8.7 & 21.96 & 0.08 & 6.8 & 0.3 & 0.235 & 32.5 & 21.60 & 7.58 & 0.33 \\
\hline $1204+24$ & 2.0 & 11.2 & 23.10 & 0.16 & 7.9 & 0.7 & 0.206 & 122.2 & 22.66 & 10.54 & 0.93 \\
\hline $1217+29$ & 2.8 & 39.1 & 20.67 & 0.06 & 28.4 & 1.0 & 0.086 & 25.2 & 20.66 & 1.15 & 0.04 \\
\hline $1251+27$ & 2.0 & 7.8 & 22.83 & 0.27 & 5.5 & 0.8 & 0.067 & 168.4 & 22.34 & 8.08 & 1.18 \\
\hline $1256+28$ & 2.0 & 6.9 & 21.64 & 0.12 & 9.5 & 0.7 & 0.077 & 163.9 & 21.51 & 3.99 & 0.29 \\
\hline $1257+28$ & 2.9 & 21.4 & 23.30 & 0.03 & 44.2 & 1.4 & 0.107 & 37.4 & 23.16 & 19.76 & 0.63 \\
\hline $1300+32$ & 2.0 & 5.8 & 22.14 & 0.42 & 3.0 & 0.6 & 0.137 & 28.3 & 21.19 & 7.61 & 1.52 \\
\hline $1316+29$ & 2.0 & 11.0 & 22.84 & 0.15 & 7.6 & 0.7 & 0.163 & 82.4 & 22.43 & 9.66 & 0.89 \\
\hline $1317+33$ & 2.0 & 13.0 & 22.24 & 0.10 & 10.0 & 0.6 & 0.038 & 24.2 & 22.02 & 6.95 & 0.42 \\
\hline $1346+26$ & 2.0 & 13.0 & 23.82 & 0.08 & 23.2 & 1.5 & 0.251 & 15.2 & 23.46 & 25.97 & 1.68 \\
\hline $1347+28$ & 2.0 & 11.7 & 23.79 & 0.15 & 12.6 & 1.3 & 0.349 & 173.1 & 23.38 & 15.93 & 1.64 \\
\hline $1422+26$ & 2.1 & 19.5 & 23.75 & 0.07 & 21.7 & 1.1 & 0.256 & 128.7 & 23.54 & 14.74 & 0.75 \\
\hline $1430+25$ & 2.0 & 7.6 & 22.88 & 0.26 & 5.9 & 0.9 & 0.430 & 164.0 & 22.42 & 8.27 & 1.26 \\
\hline $1447+27$ & 2.3 & 10.0 & 21.53 & 0.07 & 7.2 & 0.2 & 0.067 & 77.3 & 21.35 & 4.08 & 0.11 \\
\hline $1512+30$ & 2.0 & 9.4 & 22.60 & 0.12 & 7.6 & 0.5 & 0.341 & 122.8 & 22.07 & 12.01 & 0.79 \\
\hline $1527+30$ & 2.0 & 11.5 & 23.36 & 0.15 & 10.2 & 1.0 & 0.336 & 6.2 & 22.72 & 19.24 & 1.89 \\
\hline $1557+26$ & 2.0 & 11.7 & 21.98 & 0.10 & 6.9 & 0.4 & 0.149 & 89.2 & 21.73 & 5.54 & 0.32 \\
\hline $1609+31$ & 2.0 & 6.1 & 22.00 & 0.38 & 3.3 & 0.6 & 0.100 & 79.6 & 21.47 & 5.28 & 0.96 \\
\hline $1610+29$ & 2.0 & 17.7 & 21.96 & 0.04 & 14.9 & 0.3 & 0.288 & 3.2 & 21.78 & 8.63 & 0.17 \\
\hline $1615+35$ & 2.0 & 23.4 & 22.67 & 0.05 & 17.2 & 0.6 & 0.111 & 77.8 & 22.50 & 9.44 & 0.33 \\
\hline $1621+38$ & 3.8 & 42.3 & 22.78 & 0.04 & 37.1 & 1.2 & 0.484 & 174.1 & 22.60 & 21.29 & 0.69 \\
\hline $1626+39$ & 2.4 & 16.0 & 24.58 & 0.03 & 135.5 & 5.1 & 0.414 & 33.0 & 24.40 & 76.09 & 2.86 \\
\hline $1657+32$ & 2.0 & 11.5 & 22.13 & 0.13 & 6.1 & 0.4 & 0.328 & 46.9 & 21.77 & 6.81 & 0.45 \\
\hline $1658+32$ & 2.0 & 6.9 & 23.19 & 0.31 & 6.6 & 1.2 & 0.200 & 32.4 & 22.62 & 11.29 & 2.05 \\
\hline $1726+31$ & 2.0 & 4.9 & 23.54 & 0.46 & 7.0 & 1.9 & 0.416 & 87.9 & 22.57 & 18.02 & 4.89 \\
\hline $1855+37$ & 2.0 & 19.4 & 22.58 & 0.05 & 17.2 & 0.6 & 0.200 & 75.3 & 22.26 & 16.98 & 0.59 \\
\hline
\end{tabular}

prominence of bright surface brightness towards the NE. B2 $1833+32$ shows also a tail towards the East at a high surface brightness level. In addition two galaxies show extensions of low surface brightness, in the form of a tail (B2 $0222+36)$ and a shell (B2 $0258+35)$. B2 $0149+35$ is the only elliptical to clearly feature nuclear dust. The dust is revealed on the contour map in Fig. 1 and it is responsible for the flatness of the inner part of the surface brightness profile. B2 $0924+30$ may present also nuclear dust although this needs confirmation.
The galaxies B2 0116+31, B2 0120+33, B2 0648+27, and B2 1358+30 show isophote twisting, which we define by the presence of changes in the PA of the semimajor axis larger than 20 degrees per semidecade in radius. These changes were measured at distances larger than 3 arcsec to avoid the nuclear parts. B2 0222+36 and B2 1037+30 also show twists, but they could be related to the presence of the tails. The isophote twisting measured in B2 $0149+35$ is probably due to the presence of a nuclear dust lane. The galaxy B2 $1626+39$ shows also PA twist, 
Table 6. Isophotal peculiarities

\begin{tabular}{|c|c|c|c|c|c|c|c|}
\hline B2 name & Twisting & Boxy & Disky & Off-centering & Excess over $r^{1 / 4}$ & Bright nucleus & Dust \\
\hline $0116+31$ & yes & yes & yes & & & & \\
\hline $0120+33$ & yes & & & & yes & & \\
\hline $0149+35$ & yes & yes & & yes & & & yes \\
\hline $0222+36$ & yes & & yes & yes? & & & \\
\hline $0326+39$ & & & & & yes & & \\
\hline $0331+39$ & & & yes & & & & \\
\hline $0648+27$ & yes & & & & & & \\
\hline $0838+32$ & & & yes & yes & & & \\
\hline $0843+31$ & & & yes & & & & \\
\hline $0924+30$ & & & & & & & yes? \\
\hline $1003+26$ & & yes? & & & & & \\
\hline $1003+35$ & & & & yes & & & \\
\hline $1037+30$ & yes & & yes & yes & & & \\
\hline $1101+38$ & & & & & & yes & \\
\hline $1144+35$ & & yes? & & & & & \\
\hline $1204+24$ & & yes & & & & & \\
\hline $1204+34$ & & yes? & & yes & & & \\
\hline $1257+28$ & & yes? & yes? & & & & \\
\hline $1316+29$ & & & yes & & & & \\
\hline $1346+26$ & & & yes & yes & & & \\
\hline $1347+28$ & & yes? & & & & & \\
\hline $1358+30$ & yes & & yes & & & & \\
\hline $1430+25$ & & & yes? & & & & \\
\hline $1447+27$ & & & yes? & & yes & & \\
\hline $1512+30$ & & & yes? & & & & \\
\hline $1527+30$ & & & yes & & & & \\
\hline $1557+26$ & & & yes? & & & & \\
\hline $1615+35$ & & & yes? & & & & \\
\hline $1621+38$ & & yes & & & & & \\
\hline $1626+39$ & yes & yes & & yes & & & \\
\hline $1652+39$ & & & yes & & & yes & \\
\hline $1657+32$ & & & & yes & & & \\
\hline $1726+31$ & & yes & & yes & & & \\
\hline $1833+32$ & & yes & yes & & & yes & \\
\hline
\end{tabular}

Table 7. Emission line galaxies

\begin{tabular}{|c|c|c|c|c|c|}
\hline B2 name & Emission lines & BLRG & Type & Isoph. pecul. & Refs \\
\hline $0116+31$ & {$[\mathrm{O}$ II $]$} & & $\mathrm{E}$ & TI, BI, DI & 1 \\
\hline $0207+38$ & $\mathrm{H} \alpha,[\mathrm{N} \mathrm{II}]$ & & Irr & & 1 \\
\hline $0222+36$ & {$[\mathrm{O} \mathrm{II}]$} & & $\mathrm{E}$ & DI, tail, offcen & 1 \\
\hline $0258+35$ & {$[\mathrm{O}$ II $]$} & & $\mathrm{E}$ & shell & 1 \\
\hline $0648+27$ & {$[\mathrm{O} \mathrm{\text {II}}]$} & & $\mathrm{E}$ & TI, dist & 1 \\
\hline $0722+30$ & {$[\mathrm{O} \mathrm{II}],[\mathrm{O} \mathrm{III}], \mathrm{H} \alpha,[\mathrm{N} \mathrm{II}]$} & & $\mathrm{S}(\mathrm{S} 0)$ & & 1 \\
\hline $0800+24$ & {$[\mathrm{O}$ II] } & & $\mathrm{E}$ & & 1 \\
\hline $1141+37$ & [O II], [O III] & & $\mathrm{E}$ & chain of galaxies & 2 \\
\hline $1144+35$ & {$[\mathrm{O} \mathrm{II}],[\mathrm{O} \mathrm{III}]$} & & $\mathrm{E}$ & & 1 \\
\hline $1217+29$ & $\begin{array}{l}{[\mathrm{O} \mathrm{II}],[\mathrm{He} \mathrm{III}], \mathrm{H} \gamma, \mathrm{H} \beta,} \\
{[\mathrm{O} \mathrm{III}],[\mathrm{O} \mathrm{I}], \mathrm{H} \alpha,[\mathrm{N} \mathrm{II}],[\mathrm{S} \mathrm{II}]}\end{array}$ & & $\mathrm{E}$ & & 3 \\
\hline $1318+34$ & {$[\mathrm{O} \mathrm{II}]$} & & Irr & & 1 \\
\hline $1346+26$ & {$[\mathrm{O} \mathrm{II}]$} & & $\mathrm{E}$ & DI, offcen & 1 \\
\hline $1422+26$ & {$[\mathrm{O}$ II $]$} & & $\mathrm{E}$ & & 1 \\
\hline $1615+35$ & $\begin{array}{l}{[\mathrm{O} \text { II] }, \mathrm{H} \beta,[\mathrm{O} \text { III], }[\mathrm{O} \mathrm{I}], \mathrm{H} \alpha,} \\
{[\mathrm{N} \text { II] }}\end{array}$ & yes & Irr & & $4,5,6$ \\
\hline $1626+39$ & {$[\mathrm{O} \mathrm{II}]$} & & $\mathrm{E}$ & TI, BI, mult.nuc., offcen & 1 \\
\hline $1833+32$ & $\begin{array}{l}\mathrm{Ly} \alpha, \mathrm{C} \text { IV, } \mathrm{Mg} \text { II, [O II] }, \mathrm{H} \gamma, \\
\mathrm{H} \beta,\left[\mathrm{O} \text { III] }, \mathrm{H} \alpha,\left[\mathrm{N}_{\mathrm{II}}\right]\right.\end{array}$ & yes & $\mathrm{E}$ & BI, DI, dist, nucleus & 7 \\
\hline
\end{tabular}

1. Colla et al. (1975).

2. Djorgovski et al. (1990).

5. Osterbrock (1979)

3. Heckman et al. (1980).

6. Halpern (1990).

4. Burbidge \& Strittmater (1972).

7. Tadhunter et al. (1986). 

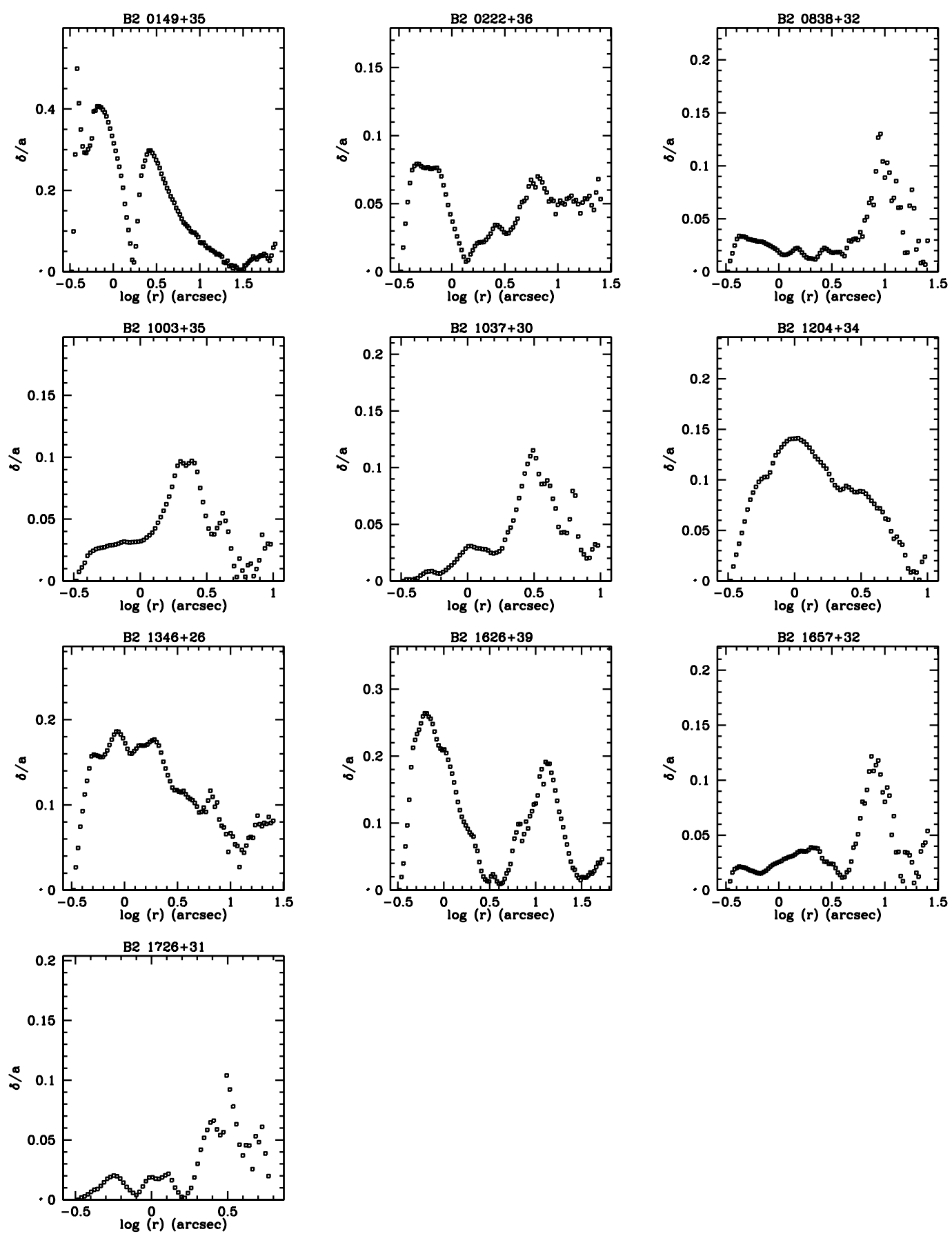

Fig. 3. $\delta / r$ deviations along semimajor axis of the galaxies exhibiting significant off-centering

but the shape of the inner isophotes could be affected by the presence of multiple nuclei in the galaxy. B2 0326+39, B2 $1113+24$, B2 $1217+29$, B2 $1257+28$ and B2 1317+33 also show strong changes in PA, but for these cases the twists are measured over isophotes with low eccentricity, where the errors in position angle are large, and thus the twisting may not be real. Furthermore the PA profile for B2 $0326+39$ and B2 $1317+33$ could be affected by nearby galaxies. Therefore only B2 116+31, B2 0120+33,
B2 $0648+27$, and B2 $1358+30$ do unambiguously reveal isophote twisting.

Another interesting parameter derived from the isophote analysis is the $B_{4}$ term of the Fourier series. This parameter should be zero for a perfect ellipse and indicates "boxy isophotes" if $B_{4}$ is negative and "disky isophotes" if $B_{4}$ is positive. We have considered as boxy (disky) those galaxies having a $B_{4}$ parameter lower (higher) than $\sim-0.02(+0.02)$ in more than 5 adjacent isophotes. 
Galaxies with noisy profiles and/or $\left|B_{4}\right|$ values in the range $0.01-0.02$ were considered as marginal cases of boxy/disky isophotes. The $B_{4}$ profiles are shown in Fig. 2. Seven galaxies of the sample appear to have boxy isophotes. The evidence for boxiness is clear for B2 $0116+31$, B2 $1204+24$, B2 $1621+38$, B2 1626+39, B2 $1726+31$, and B2 $1833+32$. B2 $0149+35$ shows a negative $B_{4}$ parameter but this is most probably due to the dust; in fact the galaxy has also a high $B_{3}$ term, which is a typical diagnostic of the presence of dust. Five other galaxies show indications of boxiness although at a marginal level.

The opposite behaviour, i.e. disky isophotes, is found for 12 galaxies which are $\mathrm{B} 20116+31$, B2 $0222+36$, B2 0331+39, B2 0838+32, B2 0843+31, B2 1037+30, B2 1316 +29, B2 1346+26, B2 1358+30, B2 1527+30, B2 1652+29 and B2 $1833+32$. Six additional galaxies are marginally disky. We note that for the galaxy B2 0838+32 several nearby nuclei/companions were masked out and this may affect the $B_{4}$ profile. For B2 $1037+30$ the isophote analysis was obtained over a rather small area of the galaxy, due to the masking of a large prominence of difusse emission and this could also affect the $B_{4}$ profile.

The separation $\delta$ between the isophote centre at semimajor axis $r$ and the centre of the inner isophote is a standard parametrization of non-concentric isophotes or off-centering, and it is an indication of the effects of tidal interactions (Paper II and references therein). Profiles of $\delta / r$ are obtained from the surface analysis method and measurement errors are around $1-5 \%$ depending on the galaxy brightness. In our sample, nine galaxies show offcentering at levels of $\sim 7-20 \%$. Two of them (B2 $0838+32$ and B2 1626+39) have multiple nuclei and B2 0149+35 has a dust lane that can be the responsible of this offcentering. Another galaxy, B2 0222+36, may have offcentering, but this needs confirmation. Profiles of the $\delta / r$ deviations for the significant cases are shown in Fig. 3.

A summary of the morphological and isophotal peculiarities of the galaxies derived from the surface photometry is given in Table 6. Cases for which evidence for a particular property is marginal are indicated by "yes?" in the corresponding column and need confirmation through deep or high-resolution imaging.

\subsection{Surface photometry of the spiral/irregular galaxies}

We have obtained the surface photometry of six galaxies classified as spirals or irregulars from the contour plots, and we present their surface brightness profiles as well as the profiles for ellipticity, position angle and $B_{4}$ in Fig. 2 . Three of the spiral galaxies (B2 0722+30, B2 0944+39 and B2 $1039+27$ ) have $B_{4}$ profiles clearly showing disky isophotes, with a high amplitude, related to the presence of disks, arms or bars. These features, responsible for the positive $B_{4}$ terms, are revealed as humps on the surface brightness profiles.

\section{Emission lines}

In Table 7 we have listed all the B2 galaxies for which the presence of emission lines is reported in the literature. Objects with emission lines represent a $\sim 22 \%$ of the sample and almost half show isophotal peculiarities, which are listed in Table 7 . The presence of broad emission lines (BLRG) is also indicated in the table.

\section{Summary}

We have obtained $V$-band images of 72 galaxies from the B2 sample, with redshifts up to 0.2 and of low radio power. Accurate $V$-band total magnitudes were obtained for all the galaxies and the presence of morphological peculiarities was analysed. The luminosity and geometrical profiles of the elliptical galaxies were determined from ellipse fitting of the isophotes and are presented in the paper. Isophote deviations such as PA twisting, boxiness, diskiness, and off-centering are studied. Most of the ellipticals follow the $r^{1 / 4}$ law, although three of them show excesses in the outer parts. In addition three B2 galaxies present a bright nucleus.

Acknowledgements. The Jacobus Kapteyn and the William Herschel Telescopes are operated by the Royal Greenwich Observatory at the Spanish Observatorio del Roque de Los Muchachos of the Instituto de Astrofísica de Canarias on behalf of the Science and Engineering Research Council of the United Kingdom and the Netherlands Organization for Scientific Research. We thank Paola Parma for useful comments on the identification of some sources. Partial financial support was provided by DGICYT project PB92-0501 (JIGS), DGES project PB95-0122 and by the Comisión Mixta Caja Cantabria- Universidad de Cantabria. This research has made use of the NASA/IPAC Extragalactic Database (NED) which is operated by the Jet Propulsion Laborartory, California Institute of Technology, under contract with the National Aeronautics and Space Administration. We are grateful to the referee, Reynier Peletier, for several suggestions on the presentation of the data.

\section{References}

Barthel P.D., Schilizzi R.T., Miley G.K., Jägers W.J., Strom R.G., 1985, A\&A 148, 243

Bridle A.H., Baum S.A., Fomalont E.B., et al., 1991, A\&A 245, 371

Burbidge E.M., Strittmater P.A., 1972, ApJ 172, L37

Burstein D., Heiles C., 1982, AJ 87, 1165

Coleman G.D., Wu C.C., Weedman D.W., 1980, ApJS 43, 393

Colina L., Pérez-Fournon I., 1990, ApJ 349, 45

Colla G., Fanti C., Fanti R., et al., 1975, A\&AS 20, 1 (C75)

Condon J.J., Cotton W.D., Greisen E.W., et al., 1998, AJ 115, 1693

Corbett E.A., Robinson A., Young S., Axon D.I., 1997, in Accretion Phenomena and Related Outflows; IAU Colloquium 163, ASP Conf. Ser. 121, Wickramasinghe D.T., Bicknell G.V., Ferrario L. (eds.), p. 693 
de Ruiter H.R., Parma P., Fanti C., Fanti R., 1986, A\&AS 65, 111

Djorgovski S., Thompson D.J., Vigotti M., Grueff G., 1990, PASP 102, 113

Douglas J.M., Bash F.N., Bozyan F.A., Torrence G.W., Wolfe C., 1996, AJ 111, 1495

Ekers R.D., Fanti R., Lari C., Parma P., 1981, A\&A 101, 194

Fanaroff B.L., Riley J.M., 1974, MNRAS 167, 31P

Fanti R., Gioia I., Lari C., Ulrich M.H., 1978, A\&AS 34, 341 (F78)

Fanti C., Fanti R., de Ruiter H.R., Parma P., 1986, A\&AS 65, 145

Fanti C., Fanti R., de Ruiter H.R., Parma P., 1987, A\&AS 69, 57 (F87)

Feretti L., Giovannini G., 1985, A\&A 147, L13

Feretti L., Giovannini G., Gregorini L., Parma P., Zamorani G., 1984, A\&A 139, 55

Giovannini G., Feretti L., Gregorini L., Parma P., 1988, A\&A 199, 73

González-Serrano J.I., Pérez-Fournon I., 1989, ApJ 338, L29 (Paper I)

González-Serrano J.I., Pérez-Fournon I., 1991, A\&A 249, 75 (Paper II)

González-Serrano J.I., Pérez-Fournon I., 1992, AJ 104, 535 (Paper III)

González-Serrano J.I., Carballo R., Pérez-Fournon I., 1993, AJ 105, 1710 (Paper IV)

Halpern J.P., 1990, ApJ 365, L51

Heckman T.M., Balick B., Crane P.C., 1980, A\&AS 40, 295

Heckman T.M., Carty T.J., Bothun G.D., 1985, ApJ 288, 122

Heckman T.M., Smith E.P., Baum S.A., et al., 1986, ApJ 311, 526

Hewitt A., Burbidge G., 1993, ApJS 87, 451

Impey C., Gregorini L., 1993, AJ 105, 853

Irwin M., 1992, Gemini 37, 1
Ledlow M.J., Owen F.N., 1995, AJ 109, 853

Lilly S.J., Prestage R.M., 1987, MNRAS 225, 531

Machalsky J., Condon J.J., 1985, AJ 90, 5

Morganti R., Fanti C., Fanti R., Parma P., de Ruiter H.R., 1987, A\&A 183, 203

Morganti R., Parma P., Capetti A., Fanti R., de Ruiter H.R., 1997, A\&A 326, 919

O'Dea C.P., Owen F.N., 1985, AJ 90, 927

Osterbrock D.E., 1979, AJ 84, 901

Owen F.N., Laing R.A., 1989, MNRAS 238, 357

Parma P., de Ruiter H.R., Fanti C., Fanti R., 1986, A\&AS 64, 135

Parma P., Fanti C., Fanti R., Morganti R., de Ruiter H.R., 1987, A\&A 181, 244

Parma P., Morganti R., Capetti A., Fanti R., de Ruiter H.R., 1993, A\&A 267, 31

Peletier R.F., Davies R.L., Illingworth G.D., Davis L.E., Cawson M., 1990, AJ 100, 1091

Roeser S., Bastian U., 1988, A\&AS 74, 449

Schilizzi R.T., Fanti C., Fanti R., Parma P., 1983, A\&A 126, 412

Schweizer F., 1998, in Galaxies: Interactions and Induced Star Formation, Friedli D., Martinet L., Pfenniger D. (eds.). Springer-Verlag, p. 105

Smith E.P., Heckman T.M., 1989a, ApJS 69, 365

Smith E.P., Heckman T.M., 1989b, ApJ 341, 658

Tadhunter C.N., Pérez E., Fosbury R.A.E., 1986, MNRAS 219, 555

van Breugel W., Jägers W., 1982, A\&AS 49, 529

van Breugel W., Heckman T.M., Miley G.K., 1984, ApJ 276, 79

van Breugel W., Miley G., Heckman T., Butcher H., Bridle A., 1985, ApJ 290, 496

White R.L., Becker R.H., Helfand D.J., Gregg M.D., 1997, ApJ 475, 479 